ARTICLE

DOI: $10.1038 / \mathrm{s} 41467-017-01987-2$

\title{
Break-induced replication promotes formation of lethal joint molecules dissolved by Srs2
}

Rajula Elango ${ }^{1}$, Ziwei Sheng ${ }^{2}$, Jessica Jackson ${ }^{3}$, Jenna DeCata ${ }^{1}$, Younis Ibrahim', Nhung T. Pham ${ }^{4}$, Diana H. Liang ${ }^{4,5}$, Cynthia J. Sakofsky ${ }^{1,6}$, Alessandro Vindigni ${ }^{3}$, Kirill S. Lobachev², Grzegorz Ira ${ }^{4}$ \& Anna Malkova iD ${ }^{1}$

Break-induced replication (BIR) is a DNA double-strand break repair pathway that leads to genomic instabilities similar to those observed in cancer. BIR proceeds by a migrating bubble where asynchrony between leading and lagging strand synthesis leads to accumulation of long single-stranded DNA (ssDNA). It remains unknown how this ssDNA is prevented from unscheduled pairing with the template, which can lead to genomic instability. Here, we propose that uncontrolled Rad51 binding to this ssDNA promotes formation of toxic joint molecules that are counteracted by Srs2. First, Srs2 dislodges Rad51 from ssDNA preventing promiscuous strand invasions. Second, it dismantles toxic intermediates that have already formed. Rare survivors in the absence of Srs2 rely on structure-specific endonucleases, Mus81 and Yen1, that resolve toxic joint-molecules. Overall, we uncover a new feature of BIR and propose that tight control of ssDNA accumulated during this process is essential to prevent its channeling into toxic structures threatening cell viability.

\footnotetext{
${ }^{1}$ Department of Biology, University of lowa, lowa City, IA 52242, USA. ${ }^{2}$ School of Biological Sciences and Institute for Bioengineering and Bioscience, Georgia Institute of Technology, Atlanta, GA 30332, USA. ${ }^{3}$ Edward A Doisy Department of Biochemistry and Molecular Biology, Saint Louis University School of Medicine, St. Louis, MO 63103, USA. ${ }^{4}$ Department of Molecular \& Human Genetics, Baylor College of Medicine, Houston, TX 77030, USA. ${ }^{5}$ Present address: Houston Methodist Hospital, 6565 Fannin St., Houston, TX 77030, USA. ${ }^{6}$ Present address: Genome Integrity and Structural Biology Laboratory, National Institute of Environmental Health Sciences, US National Institutes of Health, Research Triangle Park, Durham, NC 27709, USA. Rajula Elango and Ziwei Sheng contributed equally to this work. Correspondence and requests for materials should be addressed to

K.S.L. (email: kirill.lobachev@biology.gatech.edu) or to G.I. (email: gira@bcm.edu) or to A.M. (email: anna-malkova@uiowa.edu)
} 
D NA double-strand breaks (DSBs) is a lethal DNA damage if it remains unrepaired. Break-induced replication (BIR) is one pathway to repair DSBs where only one broken end can invade into the homologous template, which is similar to breaks formed by collapsed replication forks or eroded telomeres. BIR promotes massive genomic instability including hypermutagenesis, formation of mutation clusters and gross chromosomal rearrangements similar to those leading to cancer $^{1-6}$. Recent data obtained in human cells showed that BIR can be induced by oncogene overexpression ${ }^{7}$ or by replication stalling at chromosome fragile sites ${ }^{8}$ and can potentially serve as a trigger for carcinogenesis. In addition, $10-15 \%$ of cancer cells maintain their telomeres through alternative lengthening of telomere (ALT), a BIR-like pathway9 ${ }^{9}$. This makes it essential to understand the mechanism of BIR and to specifically identify the steps of BIR that can serve as potential targets for anti-BIR therapy.

Similar to other homologous recombination pathways, BIR initiates by $5^{\prime}-3^{\prime}$ resection of a DSB end which then invades into the homologous template and initiates synthesis that can copy $>$ $100 \mathrm{~kb}$ of the template till the end of the chromosome. Despite extensive DNA synthesis, BIR mechanism is very distinct from Sphase DNA replication. Instead of a replication fork, BIR is carried out by a migrating bubble, which leads to conservative inheritance of newly synthesized DNA ${ }^{10-12}$. Another important difference between S-phase replication and BIR is that during $\mathrm{BIR}$, leading and laggings strands are synthesized in an asynchronous manner, and this leads to accumulation of long ssDNA regions ${ }^{10}$. These regions are stabilized by RPA, ssDNA binding protein ${ }^{13}$. Another protein that binds long ssDNA accumulated in the course of BIR is Rad51, a strand exchange protein. However, it remains unknown how cells control this binding, as well as the dynamics of the resulting Rad51 nucleofilament to avoid its unscheduled pairing with a template (e.g., homologous chromosome) that can threaten genomic stability. To address this question, we decided to test the role of known Rad51 regulators in BIR.

A known regulator of Rad51 nucleofilament is Srs2 helicase. Both genetic and biochemical evidence demonstrated that Srs2 can remove Rad51 from ssDNA, an activity that limits spontaneous recombination ${ }^{14-19}$. In addition, a number of other genetic studies proposed an anti-recombination role played by Srs $2^{14-17}, 20-31$ and also by its bacterial functional homolog, $\mathrm{UvrD}^{32}$. Two domains of Srs2 are required for stripping of Rad51 from ssDNA (so called "strippase" activity): the translocase and Rad51-interacting domains. The translocase (motor) domain of Srs2 resides in N- terminal portion of the protein and can be disrupted by a K41A mutation, inactivating ATPase activity of the protein $^{33}$. The Rad51-interaction (BRC) domain resides in the Cterminal part of Srs2 and is disrupted in srs2-BRCA lacking $862-914$ residues $^{34}$. Another biochemical function of Srs2 is in its helicase activity. This activity can unwind various DNA substrates $^{35-38}$, but the role of the helicase activity for the antirecombination function of Srs2 remains unclear.

We note that previous work suggested a pro-recombinogenic role for Srs2 in DSB repair pathways via homologous recombination (HR). Srs2 was reported to promote DSB repair by synthesis-dependent strand annealing (SDSA) and to limit crossover outcomes $^{39}, 40$. Also, Srs2 was proposed to be involved in DNA damage checkpoint recovery ${ }^{41,42}$, and this function was deduced from the observation of massive cell death following completion of DSB repair. In the most extreme case, $98 \%$ of $s r s 2 \Delta$ cells died despite of what appeared as successful completion of DSB repair by single-strand annealing (SSA), as detected by Southern blot analysis ${ }^{41}$. Later, this death was attributed to the incomplete unloading of recombination factors leading to persistent binding of Rad51 and RPA to the ssDNA surrounding the areas of repair even after the repair has been completed ${ }^{43}, 44$ Together these studies proposed different pro-recombination roles of Srs2 in DSB repair.

In this study, we provide the evidence for a different "antitoxic" role Srs2 plays to ensure successful DSB repair. Here, we report that bubble migration during BIR requires Srs2. We demonstrate that in the absence of Srs2, most cells fail to complete BIR and die. This massive death results from accumulation of unresolved toxic joint molecules that are formed by invasion of long ssDNA into the intact donor, which leads to trapping of donor and recipient chromosomes together and interferes with BIR completion. We propose that two main activities of Srs2, strippase and helicase, counteract toxic joint intermediates by preventing their formation and promoting their disruption, respectively. Overall, our findings demonstrate that ssDNA produced by bubble-migration represents a vulnerable intermediate of BIR that could be lethal for the cell, and we speculate that this property can be used in the future to specifically target cells undergoing BIR.

\section{Results}

Srs2 counteracts toxic joint molecules formed during BIR. To examine whether BIR promotes the formation of toxic recombination intermediates in the absence of Srs2, we used a budding yeast experimental system where a DSB is repaired by BIR involving homologous chromosomes ${ }^{45}$ (Fig. 1a, Supplementary Table 1). In this system, a galactose-inducible DSB is initiated by $\mathrm{HO}$ endonuclease at the MATa locus of the truncated copy of chromosome III (Chr. III), while the full-length donor copy of

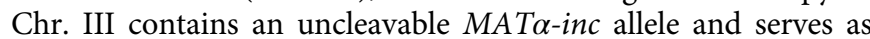
the template for DSB repair. Elimination of all but $46 \mathrm{bp}$ of homology on one side of the break on the recipient molecule via replacement with LEU2 and telomere sequences results in efficient DSB repair through BIR. Initiation of BIR in this system is preceded by extensive $5^{\prime}$-to- $3^{\prime}$ resection of the DSB at $M A T a^{46}$, and is followed by strand invasion of the $3^{\prime}$ single-strand end into the donor chromosome at a position proximal to the $\mathrm{Y} \alpha$-inc sequences and the subsequent copying of $\sim 100 \mathrm{~kb}$ of donor DNA to the right telomere (Fig. 1a). BIR and alternative repair outcomes can be followed based on maintenance of markers located at all arms of recombining molecules (Fig. 1b).

Here, using this BIR assay, we observed that $\operatorname{srs} 2 \Delta$ mutant cells exhibited a large loss of viability (Fig. 1c), while BIR remained the predominant repair outcome among srs $2 \Delta$ survivors (Fig. 1d). BIR events obtained in $s r s 2 \Delta$, however, differed from those in wild- type (SRS2) cells with respect to mutagenesis that is normally high in association with $\mathrm{BIR}^{47}$. The level of frameshifts measured using lys $2-A_{4}$ reporters placed on the track of BIR was reduced five-fold and four-fold for $16 \mathrm{~kb}$ and $36 \mathrm{~kb}$ positions of the reporter, respectively, in $s r s 2 \Delta$ as compared to wild type (SRS2) cells (Supplementary Fig. 1a). The level of base substitutions, measured using ura3-29 reporter (similar to ref. ${ }^{10}$ ) was also reduced five-fold and seven-fold for two different orientations of the reporter (Supplementary Fig. 1b). This reduced mutagenesis suggested that the absence of Srs2 affected BIR progression among the survivors as well.

Massive death observed in $s r s 2 \Delta$ mutants was atypical for our experimental system, where the presence of two copies of chromosome III, one of which remains unbroken, allows the cells to survive even in situations when a second, broken chromosome is unrepaired and lost (Fig. 1b), e.g., in rad52A mutants ${ }^{48}$. Thus, the loss of viability suggested that initiation of BIR in the absence of Srs2 compromises not only the broken chromosome but also the intact donor serving as a template for 
a

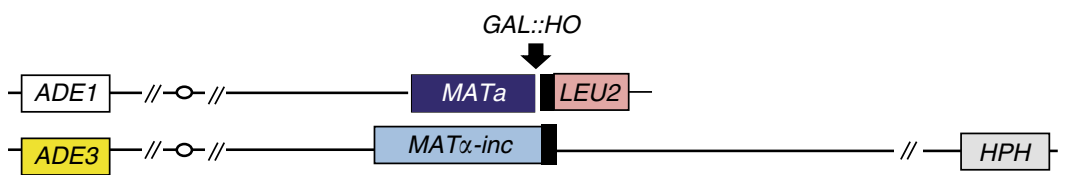

b

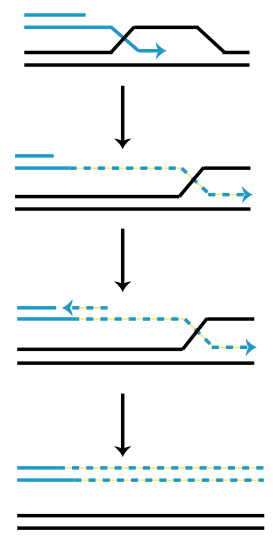

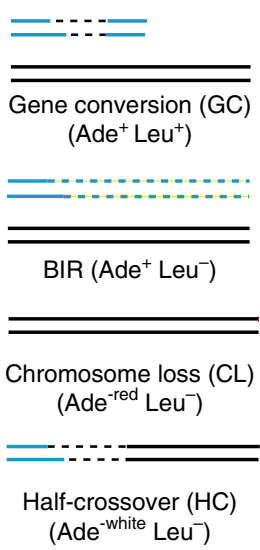

e $\begin{array}{lllll}0 & 4 & 6 & 8 & 10\end{array}$
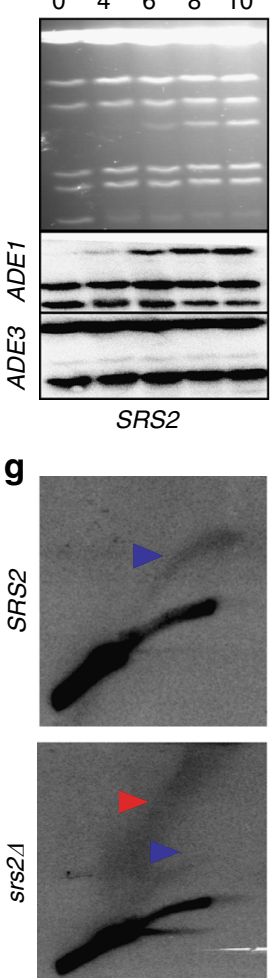

$0 \mathrm{~kb}$

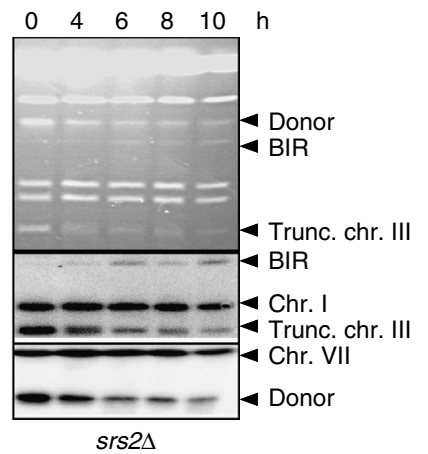

$\operatorname{srs} 2 \Delta$
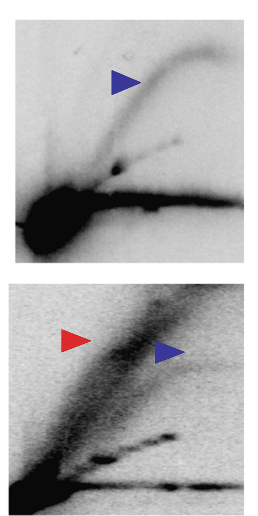

$24 \mathrm{~kb}$
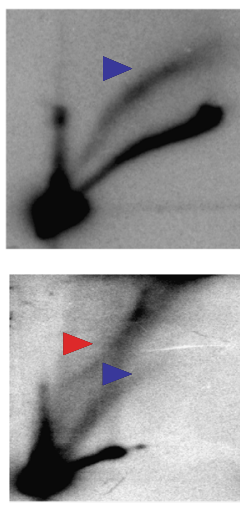

$85 \mathrm{~kb}$
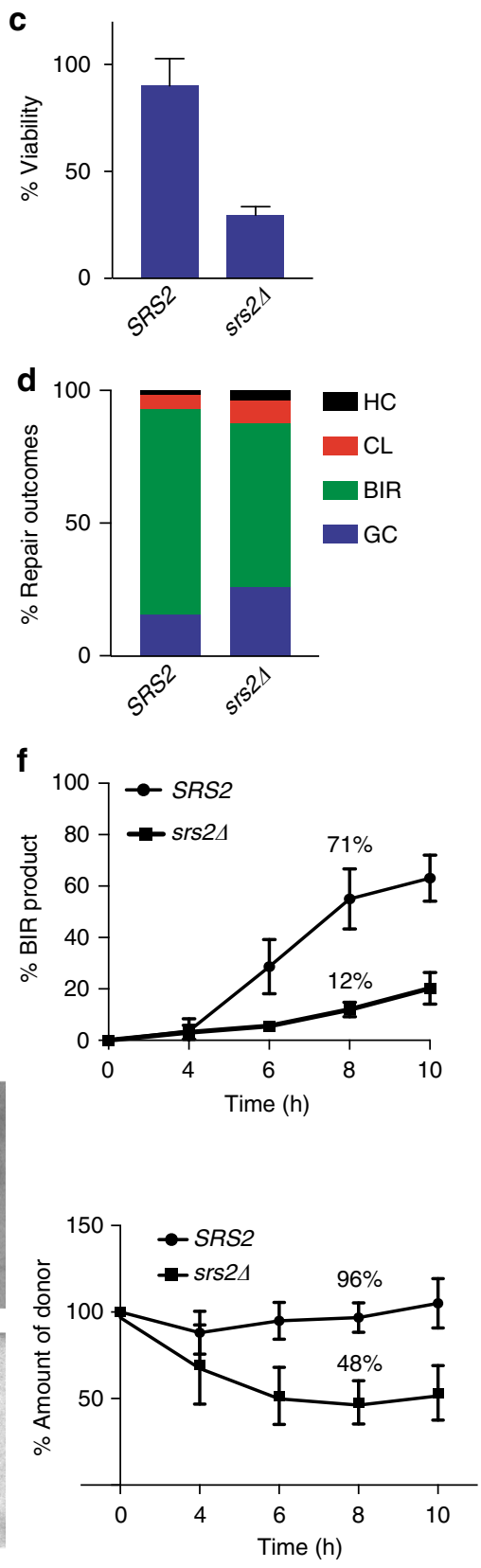

Fig. 1 BIR leads to the formation of toxic joint molecules counteracted by Srs2. a BIR is initiated by DSB introduced by HO endonuclease at MATa locus in yeast disomic for $\mathrm{Chr}$. III. The broken recipient chromosome (blue) invades unbroken homologous donor (black). Repair DNA synthesis is initiated and progresses by a migrating bubble, which leads to the conservative inheritance of newly synthesized DNA. b DSB repair outcomes with corresponding phenotypes (in parenthesis). c Cell viability following DSB induction (\%). d Distribution of DSB repair outcomes in SRS2 (WT) and srs2 $\Delta$ based on their phenotypes (refer to (b)). e BIR kinetics analyzed by CHEF gel using cells taken at indicated time points following DSB induction. Upper panel: CHEF gels stained with Ethidium Bromide. Subsequent panels below show Southern blot analysis using ADE1-specific, and ADE3-specific probes as indicated. $\mathbf{f}$ Quantification of BIR product (top) and of donor chromosome entering the gel (bottom). $\mathbf{g}$ 2D gel analysis of BIR intermediates in SRS2 (top panel) and srs2 2 (bottom panel) at $7 \mathrm{~h}$ following DSB induction. Genomic DNA was digested with Kpnl to detect intermediates at $0 \mathrm{~kb}$ and with Bglll to detect intermediates at $24 \mathrm{~kb}$ and $85 \mathrm{~kb}$. Intermediates were detected using probes specific to the following positions on Chr. III: $0 \mathrm{~kb}$ (left panel), $24 \mathrm{~kb}$ (middle panel) and $85 \mathrm{~kb}$ (right panel) away from the DSB. Blue arrowheads denote bubble arc intermediate and red arrowheads denote 'rubble' structure. Quantification of cell viability and DSB repair efficiency was based on results of $\geq 3$ independent experiments and are presented as means \pm s.d 
a

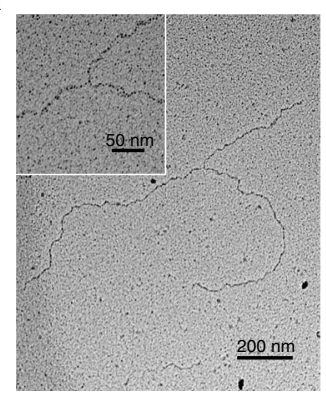

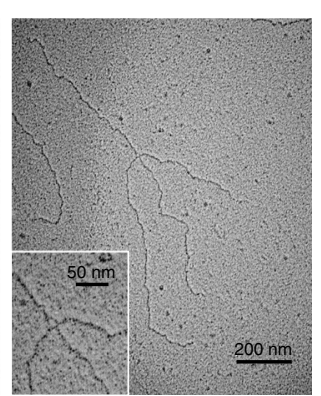

b

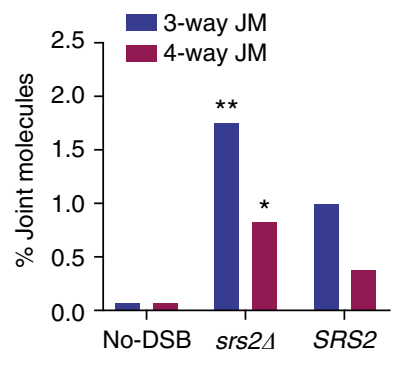

C

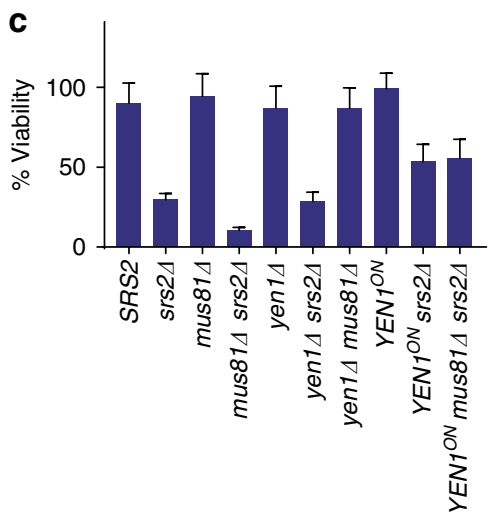

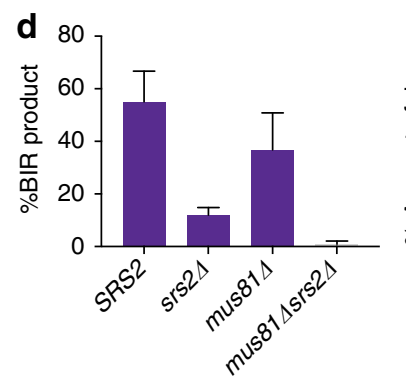

$\mathbf{f}$

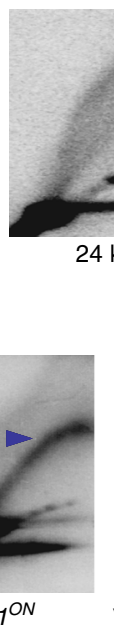

i

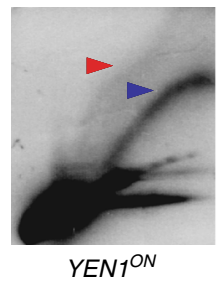

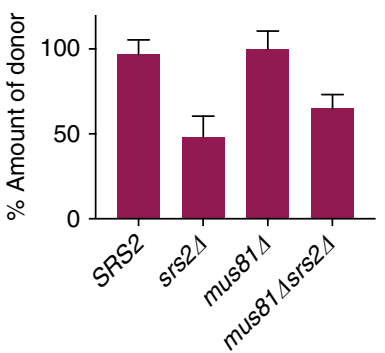

g

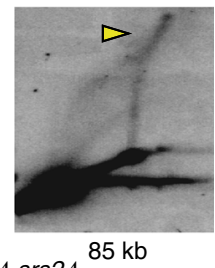

mus814 srs24

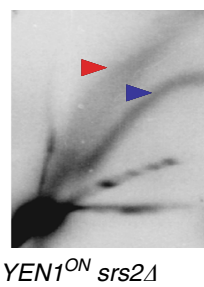

j

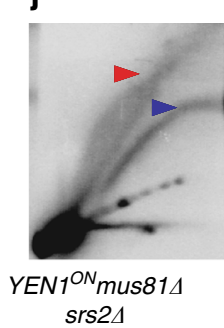

i্

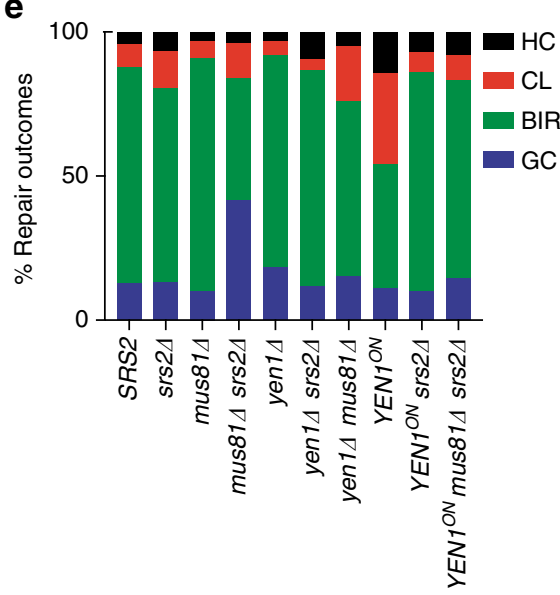

Fig. 2 Structure-specific endonucleases resolve toxic BIR intermediates. a Representative images of DNA fragments containing 3-way and 4-way junctions (joint molecules, JMs) in srs $2 \Delta$ identified by EM analysis of genomic DNA isolated $7 \mathrm{~h}$ following induction of BIR. Scale bars correspond to $200 \mathrm{~nm}$ in the entire field images and $50 \mathrm{~nm}$ in the enlarged images. b The fraction of DNA fragments containing 3-way and 4-way junctions in SRS2, srs2 4 and no-DSB srs $2 \Delta$ control. The ** and * indicate a statistically significant difference from SRS2 with $P=0.0066$ and $P=0.0379$, respectively. c Cell viability following BIR induction in cells containing various mutations, including srs $2 \Delta$, mus $81 \Delta$, yen $1 \Delta$, and YEN1 ${ }^{\mathrm{ON}}$. d Quantification of BIR product (left) and of the donor chromosome (right) at $8 \mathrm{~h}$ following initiation of BIR. e Distribution of DSB repair outcomes following BIR repair in various mutants shown in C. $\mathbf{f}$ and $\mathbf{g} 2 \mathrm{D}$ gel analysis of BIR intermediates in srs $2 \Delta$ mus $81 \Delta$ using probes specific to $24 \mathrm{~kb}$ position and $85 \mathrm{~kb}$ position, respectively, away from the DSB site. $\mathbf{h}$-j shows $2 \mathrm{D}$ intermediates detected by $24 \mathrm{~kb}$ probe in $\mathbf{h}$ YEN1 ${ }^{\mathrm{ON}}, \mathbf{i}$ YEN1 ${ }^{\mathrm{ON}}$ srs $2 \Delta, \mathbf{j}$ YEN1 ${ }^{\mathrm{ON}}$ mus $81 \Delta$ srs $2 \Delta$. Yellow and blue arrowheads denote "spike" and bubble intermediates, respectively. Red arrowhead denotes 'rubble' intermediate. Quantification of cell viability, the amount of product formed, and amount of donor present was based on $\geq 3$ independent experiments and presented as means with standard deviations (s.d.)

repair. To test this idea we analyzed BIR progression using chromosome-separating CHEF gel electrophoresis and observed two striking defects in $\operatorname{srs} 2 \Delta$. First, the amount of BIR product measured 8 hours (h) after DSB induction was nearly five-fold less than in wild-type (Fig. 1e, f). Second, while the amount of template (donor) Chr. III molecule in wild-type cells remained constant throughout the course of BIR, in srs $2 \Delta$ it drastically decreased. At $8 \mathrm{~h}$, the amount of the donor entering the gel was only $48 \%$ of the initial amount before DSB induction in $s r s 2 \Delta$ as compared to $96 \%$ in SRS2 (Fig. 1e, f).
We hypothesized that the decrease of donor molecules in the agarose gel in srs $2 \Delta$ results from accumulation of recombination intermediates as branched DNA structures. This was tested using 2D gel electrophoresis of restriction enzyme digested genomic DNA obtained from SRS2 and srs $2 \Delta$ cells undergoing BIR. We have previously used this method to demonstrate that BIR is carried out by a migrating bubble, and ssDNA accumulates due to asynchronous synthesis of leading and lagging strands ${ }^{10}$. Following 2D gel electrophoresis, DNA digested with BglII or KpnI (see legend to Fig. 1g) was hybridized with radioactively labeled 
probes specific to various positions located $0 \mathrm{~kb}, 24 \mathrm{~kb}$ and $85 \mathrm{~kb}$ centromere-distal to the DSB position (Fig. 1g). BIR in wild-type cells was associated with the formation of bubble-arc replication intermediate that was previously described ${ }^{10}$. In $s r s 2 \Delta$, the bubble intermediate (blue arrow) was barely detectable while another BIR intermediate became more prominent. This intermediate a
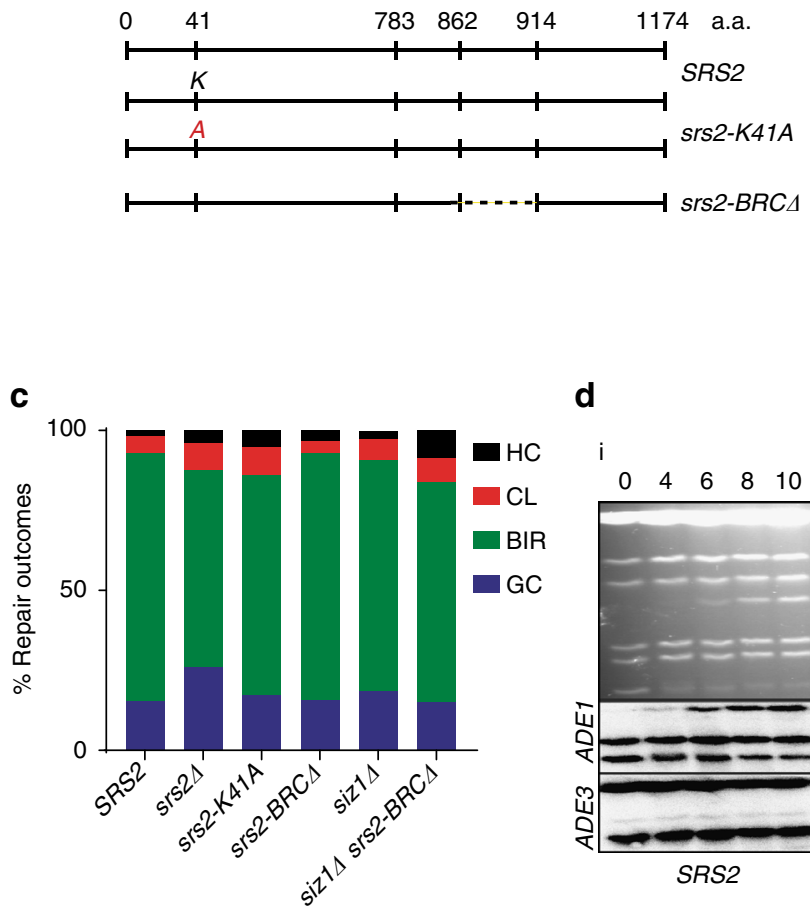

d

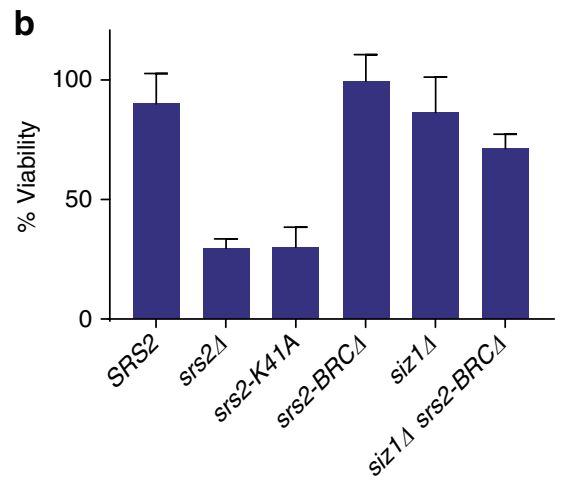

e
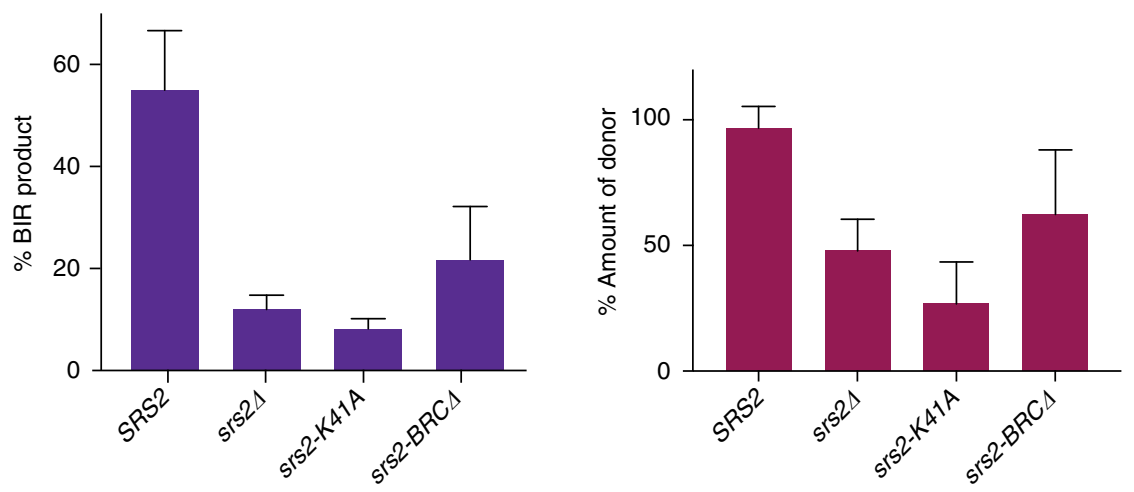

f

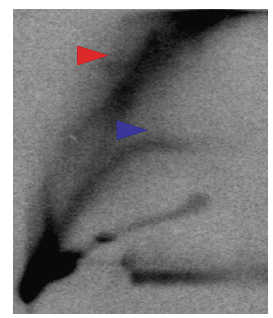

srs2-K41A g

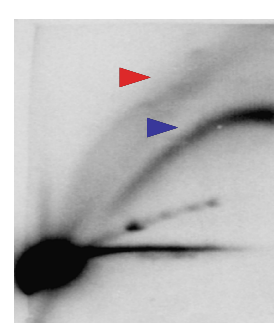

$\operatorname{srs} 2-B R C A$

Fig. 3 The defects in the helicase and strippase activities of Srs2 interfere with BIR progression. a The schematic of Srs2 (WT) and of srs2 mutations srs2$K 41 A$ and srs2-BRCA. "A" represents the position where lysine is replaced by alanine resulting in the ATPase (strippase and helicase) defect. Dotted line denotes deletion of SRS2 BRC domain. b Cell viability following DSB induction in SRS2, srs2 $\Delta$, srs2-K41A, srs2-BRCA, siz1 and siz1 srs2-BRC $\Delta$. c Distribution of DSB repair outcomes following BIR repair in various mutants shown in $\mathbf{b}$. d BIR kinetics in srs2-K41A and srs2-BRC $\Delta$ analyzed by CHEF (see Fig. 1e for details). e Quantification of BIR product (left) and of donor entering the gel (right) measured at $8 \mathrm{~h}$ after DSB. $\mathbf{f}, \mathbf{g} 2 \mathrm{D}$ analysis of BIR in srs2-K41A and srs2$B R C \Delta$, respectively A probe specific to $24 \mathrm{~kb}$ away from the DSB was used. Blue and red arrowheads denote bubble arc intermediate and 'rubble' structure respectively. Quantification of cell viability, amount of BIR product, and amount of donor was based on $\geq 3$ independent experiments and are presented as means with standard deviations (s.d.) 

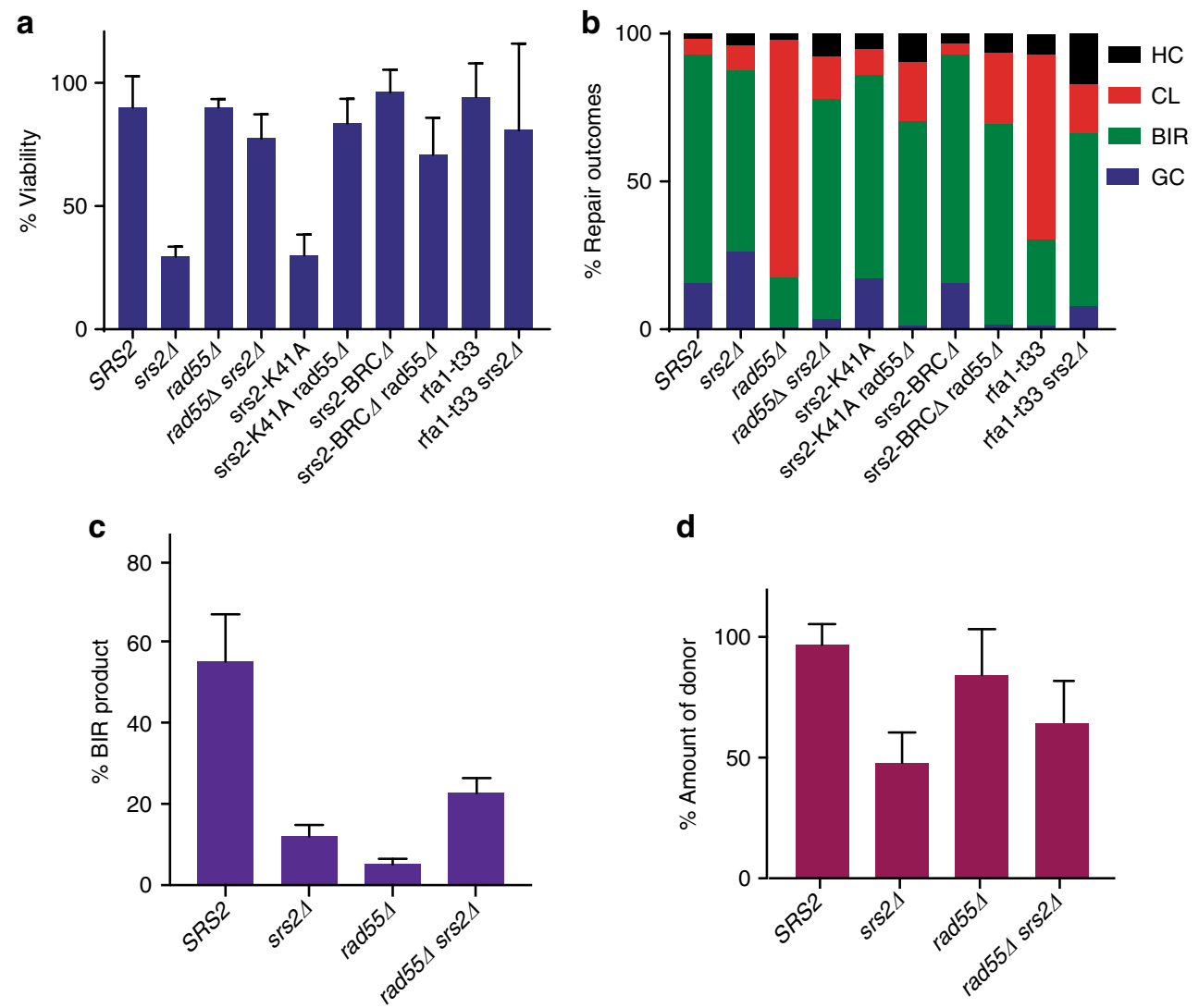

d

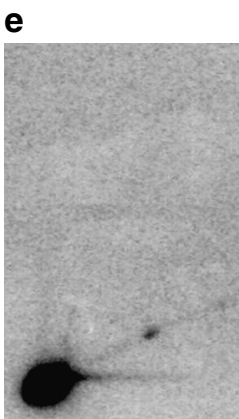

$\operatorname{rad554}$ f

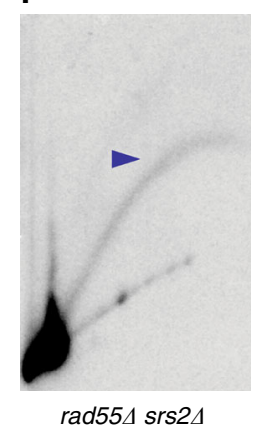

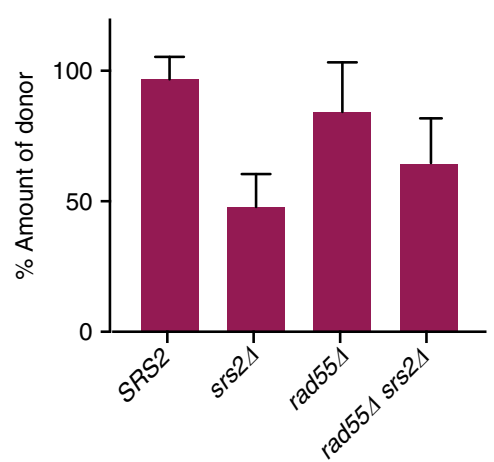

g

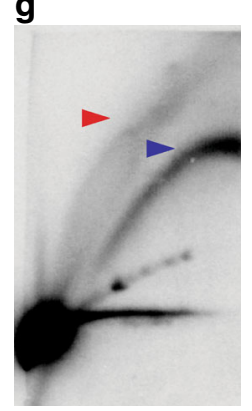

$\operatorname{srs} 2-B R C \triangle$ h

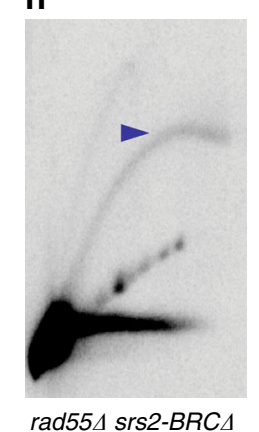

Fig. 4 Destabilization of Rad51 filament bypasses the requirement for Srs2 during BIR. a Cell viability following DSB induction in rad55 mutants. $\mathbf{b}$ distribution of repair outcomes following DSB induction in rad554, rfa1-t33 and srs $2 \Delta$ mutants. Quantification of BIR product $\mathbf{c}$ and of the donor entering the gel $\mathbf{d}$ measured at $8 \mathrm{~h}$ following DSB repair initiation. e-h 2D gel analysis of BIR intermediates in the following rad55 and srs2 mutants: e rad55 f rad55 s srs $2 \Delta, \mathbf{g}$ srs2-BRC $\Delta$, and $\mathbf{h}$ rad55 $\operatorname{srs} 2-B R C \Delta$. Blue and red arrowheads denote bubble arc intermediate and "rubble" structure respectively. Quantification of cell viability, amount of BIR product, and amount of donor was based on $\geq 3$ independent experiments and are presented as means with standard deviations (s.d.)

(Fig. 1g; red arrow) consisted of heterogeneous DNA molecules that were more branched and heavier than those forming the bubble intermediate and we will refer to this intermediate as 'the rubble'.

We propose that long stretches of ssDNA generated by DSB resection and DNA synthesis can promote formation of additional joint structures located behind the migrating BIR bubble and interfere with BIR completion. This hypothesis of joint molecule formation was tested by electron microscopy (EM) analysis of whole genome from DNA extracted from $s r s 2 \Delta$ and SRS2 cells, digested with BglII and enriched for ssDNAcontaining fragments by passing through a BND cellulose column (Fig. 2a, b; Supplementary Fig. 2 and 3). We observed a significant increase $(P=0.0003)$ of branched DNA molecules, which included 4-way and 3-way junctions in srs $2 \Delta(100 / 3860$ molecules) as compared to SRS2 cells (47/3395 molecules) (Supplementary Table 2). This was consistent with accumulation of joint molecules (JMs) in srs $2 \Delta$. As expected, the level of branched molecules was very low in a no-DSB control (Fig. 2b).

BIR toxic JMs are resolved by structure-specific nucleases. Based on the large amount of unusual joint molecules observed during BIR in srs $2 \Delta$ cells, we hypothesized that DSB repair survivors in $s r s 2 \Delta$ cells may require nucleases that cleave JMs. Towards this goal we tested the effect of elimination of Mus81Mms4 from srs $2 \Delta$ cells and activation of Yen1 that is normally inactive in G2. Both Mus81 and Yen1 are known to process 
Holliday junctions $(\mathrm{HJ})$, and other branched intermediates (reviewed in ref. ${ }^{49}$ ). Elimination of Mus 81 in $s r s 2 \Delta$ led to further decrease of cell viability upon DSB induction: from $30 \%$ in $s r s 2 \Delta$ to only $10 \%$ in $s r s 2 \Delta$ mus $81 \Delta$ (Fig. 2c). Deletion of MUS81 itself does not affect repair or viability following BIR induction ${ }^{12}$ (Fig. 2c and Supplementary Fig. 4). In addition, the amount of BIR product accumulated in srs $2 \Delta m u s 81 \Delta$ at $8 \mathrm{~h}$ following the break (as detected by CHEF) was 10 -fold lower as compared to srs $2 \Delta$ (Fig. 2d). Based on these results, we propose that Mus81 resolves some of the toxic JMs accumulated in the absence of Srs2. In support of this conclusion, we found that the structures observed by $2 \mathrm{D}$ gel electrophoresis of BIR intermediates accumulated in $s r s 2 \Delta$ mus $81 \Delta$ looked different from the 'rubble' structures. In particular, the intermediates accumulated in $s r s 2 \Delta$ $m u s 81 \Delta$, contained molecules of larger mass than those in the "rubble" structure (Fig. 2f, g; yellow arrows) and were reminiscent of the 'spike' structures that were previously reported for joint molecules in meiosis ${ }^{50-52}$. We propose that these structures represent a high molecular weight and highly branched intermediate corresponding to the multi-invasion regions formed behind the bubble (See Supplementary Fig. 5 and discussion for details). We envision that partial resolution of these multiinvasion intermediates by Mus81 leads to formation of transient intermediates (Supplementary Fig. 6) that are visualized as rubbles on 2D gel electrophoresis. These transient intermediates can be processed further which allows formation of viable BIR outcomes.

The role of another structure-specific endonuclease Yen1 was not evident based on the viability data of yen $1 \Delta$ srs $2 \Delta$ double mutant (Fig. 2c). This is expected because Yen1 nuclease is activated only in late M-phase ${ }^{53,54}$. In order to test the ability of Yen1 to resolve toxic DNA structures formed in $s r s 2 \Delta$, we used a constitutively active allele $Y E N 1^{O N 53}$. We observed that expression of $Y E N 1^{O N}$ largely suppressed the defect of both srs $2 \Delta$ and srs $2 \Delta$ mus $81 \Delta$ mutant cells, which suggested that $\mathrm{Yen}^{\mathrm{ON}}$ is capable of processing toxic intermediates formed in these mutants and the resolution of these intermediates helps survival (Fig. 2c). Consistently, YEN1 ${ }^{O N} \operatorname{srs} 2 \Delta$ mus $81 \Delta$ accumulated a "rubble" intermediate (similar to the one observed in $\operatorname{srs} 2 \Delta$ ) instead of a 'spike' structure that was observed in $\operatorname{srs} 2 \Delta$ mus $81 \Delta$ (Fig. 2h-j). Interestingly, expression of $Y E N 1^{O N}$ in wild-type cells resulted in decreased BIR and an increase in chromosome loss (CL) and half-crossover (HC) events (Fig. 2e; 1b). However, the formation of $\mathrm{CL}$ and $\mathrm{HC}$ events were very rare following BIR initiation in YEN1 ${ }^{O N}$ srs $2 \Delta$ strains (Fig. 2e). We note that the abnormal repair observed in SRS2 YEN1 ${ }^{O N}$ cells is in agreement with the view that Yen 1 should be normally inactive at G2 when BIR occurs. In addition, using the same strains, we observed that YEN1 ${ }^{O N}$ partially suppressed MMS sensitivity of srs2D (Supplementary Fig. 7a), which suggested that the molecular function of Srs2 that we discovered for BIR might be involved in repair of MMS-induced DNA damage as well.

Two detox functions of Srs2 during BIR. We propose that Srs2 promotes BIR by counteracting toxic intermediates via its two anti-recombinational activities: a "strippase" activity, which removes Rad51 from ssDNA thus preventing joint molecule formation and also through its helicase activity (reviewed in ref. ${ }^{55}$ ) which physically disrupts the toxic joint intermediates. To test this idea we examined the effects of mutations in two Srs2 domains: (i) the ATPase domain (srs2-K41A) required for stripping Rad51 and DNA unwinding ${ }^{33}$; and (ii) the BRC-domain (srs2-BRCA) required for $\operatorname{Rad} 51$ interaction and for efficient stripping of Rad51 from ssDNA ${ }^{34}$ (Fig. 3a). The effect of srs2$K 41 A$ mutation on BIR was similar to that of $s r s 2 \Delta$ with respect to cell survival (Fig. 3b), and the amount of BIR product formation (Fig. 3d, e). In addition, similar to $s r s 2 \Delta$, a large fraction of the srs2-K41A donor molecules failed to enter the CHEF gel following DSB induction (Fig. 3d, e), and accumulated 'rubble' intermediates as monitored by $2 \mathrm{D}$ gel electrophoresis (Fig. 3f). Thus, the ATPase activity of Srs2, which is required for both its strippase and helicase activities, is necessary for BIR.

Next, we tested the importance of the BRC domain of Srs2 (encompassing residues 862-914 (Fig. 3a)) in BIR. We expected that the absence of this region would affect the ability of Srs2 to remove Rad51 from the filament, but not its helicase activity ${ }^{34,35}$. We proved this for our strain background by confirming that srs2-BRCA suppressed the MMS sensitivity of rad18 $\Delta$ (Supplementary Fig. 7b) similar to other srs 2 mutants that were deficient in the "strippase" activity but possessed helicase activity ${ }^{34}$. The BIR assay performed in srs2-BRCA demonstrated successful BIR completion with the viability of $\sim 90 \%$ (Figs. $3 \mathrm{~b}$ and $4 \mathrm{a}$ ), and with BIR outcomes comprising $80 \%$ of the survivors (Fig. $4 \mathrm{~b}$ ). At the same time, the physical analysis of BIR using CHEF indicated slower kinetics of BIR with only $22 \%$ of BIR product accumulated in $s r s 2-B R C \Delta$ at $8 \mathrm{~h}$ vs. $55 \%$ in SRS2 cells (Fig. $3 \mathrm{~d}, \mathrm{e}$ ). Also, a significant fraction of the donor failed to enter the agarose gel during CHEF analysis in the middle of BIR time course (Fig. 3d, e). These defects were indicative of branched structure accumulation. Indeed, 2D gel electrophoresis detected "rubble" intermediates in srs2-BRCA cells (Fig. 3g). Based on these results we propose that the defect of $s r s 2-B R C \Delta$ in removal of the Rad51 from the filament ${ }^{34}$ leads to the formation of toxic intermediates that are eventually removed by the helicase activity of Srs2, allowing slower but efficient repair and survival.

Unstable Rad51 filament bypasses the need for Srs2. We hypothesized that the need for Srs 2 could be bypassed by having less stable Rad51 nucleofilament. Towards this goal, we used the rad55 $\Delta$ mutant where Rad51 filament is inherently unstable ${ }^{30}$. Deletion of RAD55 in srs $2 \Delta$ led to complete recovery of BIR and cell viability (Fig. $4 \mathrm{a}, \mathrm{b}$ ). The viability of $s r s 2 \Delta$ rad55 $\Delta$ cells was $80 \%$, which is much higher than in $s r s 2 \Delta(30 \%)$, and $75 \%$ of the survivors in srs $2 \Delta$ rad55 $\Delta$ resulted from BIR, which was significantly higher compared to rad55 single mutant (17\%). The latter observation suggests that Srs2-dependent removal of Rad51 in the absence of Rad55 prevents successful strand invasion, which leads to chromosome loss.

Similarly, combining rad55 $\Delta$ and the helicase-dead mutant, srs $2-K 41 A$, led to high cell viability (to $\sim 80 \%$ ) and efficient $(\sim$ $70 \%$ ) BIR (Fig. 4a, b). This result was further confirmed by $2 \mathrm{D}$ gel electrophoresis, where normal BIR bubble-arc, and no "rubble"

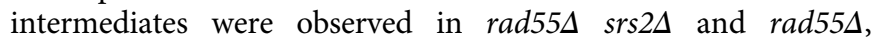
respectively (Fig. 4f, e). These results suggest that when the formation of toxic intermediates is precluded by instability of Rad51 filament, Srs2 is less needed for the completion of BIR than in the case of "excessive" and stable Rad51 filament. While the efficiency of BIR in srs $2 \Delta$ rad55 $\Delta$ mutants appears to be much better than in single $s r s 2 \Delta$ or rad55 $\Delta$ mutants, the kinetics of BIR product formation in $s r s 2 \Delta$ rad55 $\Delta$ is significantly slower than in wild-type cells, with respectively $23 \%$ vs. $55 \%$ of product accumulated $8 \mathrm{~h}$ following DSB induction (Fig. 4c). This slower BIR kinetics in srs $2 \Delta$ rad55 $\Delta$ indicates that the maximum efficiency of BIR requires optimized Rad51 filament that can be formed only in the presence of both Srs2 and Rad55. Nevertheless, most srs $2 \Delta$ rad55 $\Delta$ cells successfully completed BIR. In addition, the srs2-BRC $\Delta$ allele restored BIR in rad55 $\Delta$ mutants (Fig. $4 \mathrm{a}, \mathrm{b}, \mathrm{h}$ ), but the extent of restoration was less as compared to $\operatorname{srs} 2 \Delta$ (Fig. $4 \mathrm{~b}$ ). We envision that the higher frequency of chromosome losses observed in srs2-BRCA rad55 $\Delta$ likely resulted 
a

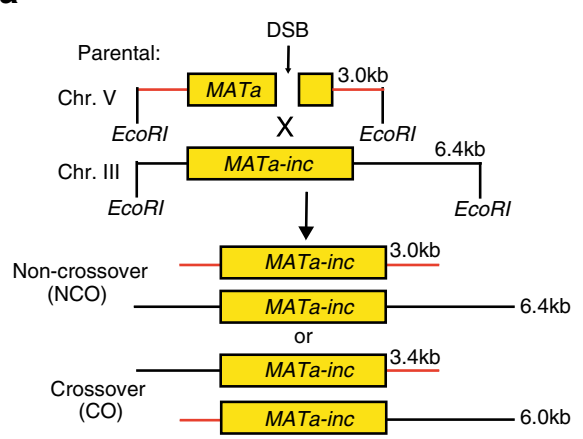

b

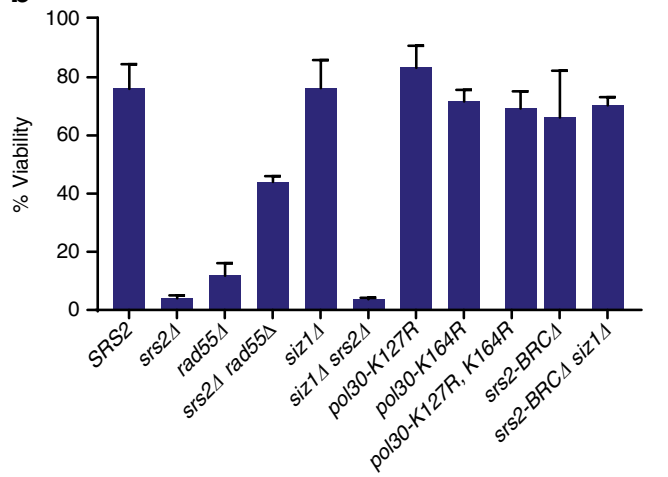

C
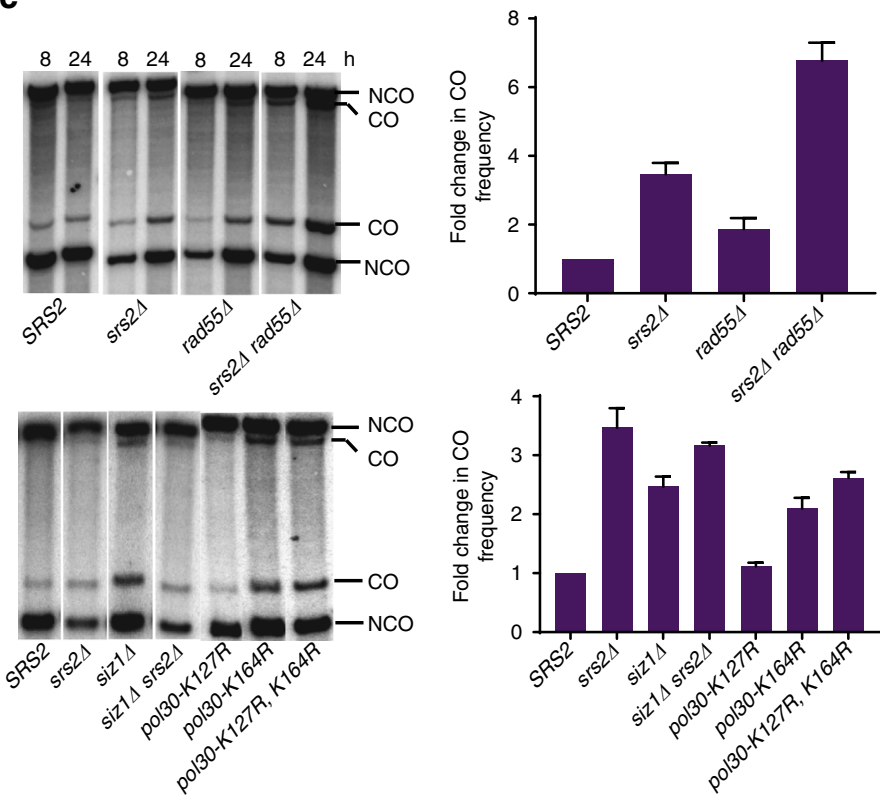

d
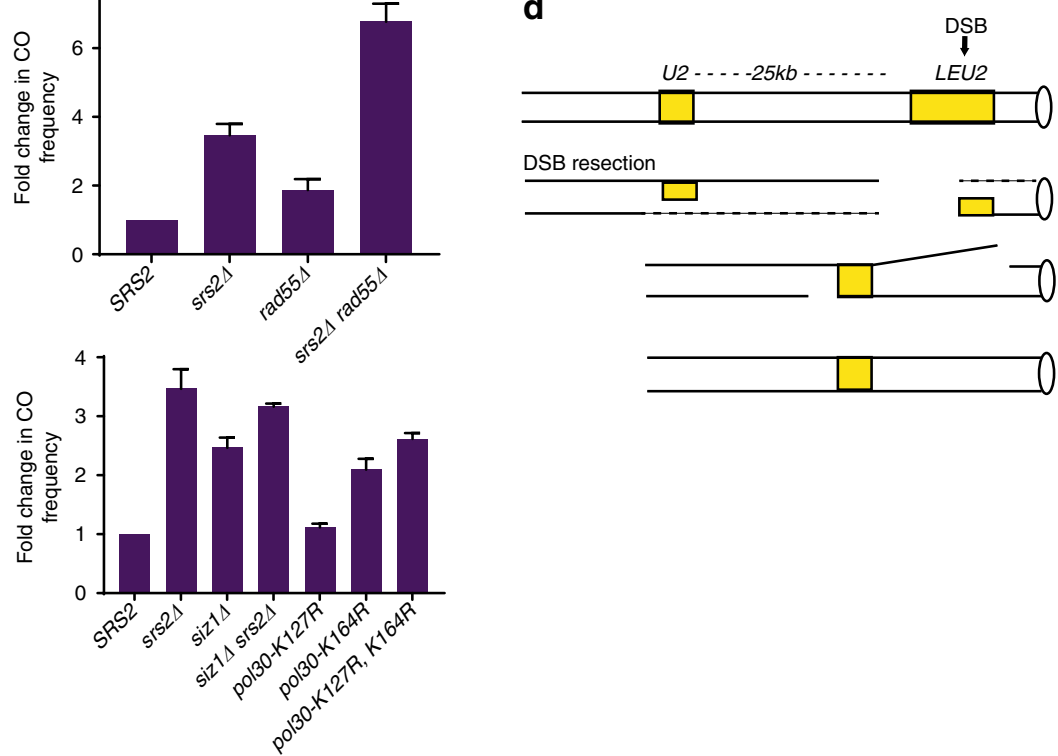

e

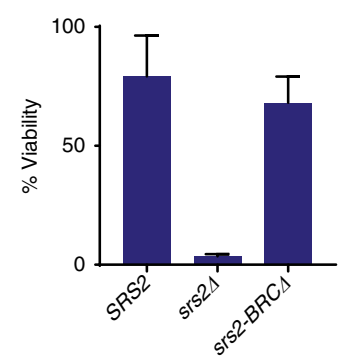

$\mathbf{f}$

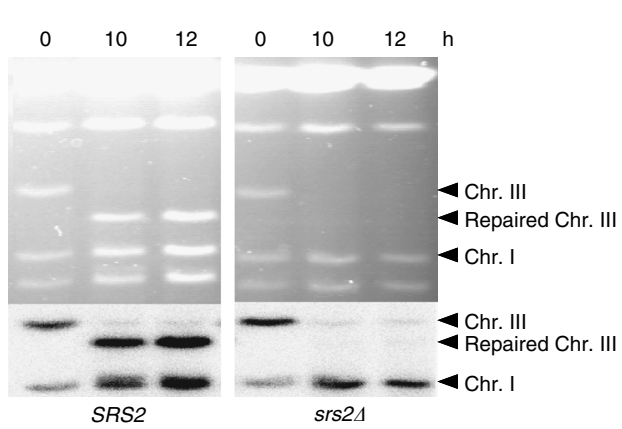

Fig. 5 Anti-toxic function of Srs2 during various DSB repair pathways. a Ectopic gene conversion is initiated by DSB introduced by HO endonuclease at MATa inserted at the ARG5,6 locus on Chr. V and proceeds by using homologous MATa-inc sequence on Chr. III as a template. The schematic explains the assay to distinguish crossover from non-crossover products of GC. b Cell viability reflecting the efficiency of GC in various mutant backgrounds. c Crossover (CO) and non-crossover (NCO) GC products detected by Southern blot analysis (left, top panel) at $8 \mathrm{~h}$ and $24 \mathrm{~h}$ for SRS2, srs2 24 , rad554, and rad55 srs2 2 . CO and NCO GC products were also detected at $8 \mathrm{~h}$ for various other mutants (left, bottom panel). $\mathbf{d}$ The schematic of DSB repair system in YMV80 strains where DSB is induced at LEU2 by HO endonuclease on Chr.III and repaired by SSA/BIR using U2 as a template. e Cell viability in SRS2 and srs2 mutants following SSA/BIR. $\mathbf{f}$ CHEF gels showing analysis of repair by SSA/BIR in SRS2 (wild type; left) and srs2 (right). Upper panel: Ethidium Bromide stained gel. Lower panel: Southern blot analysis using ADE1-specific probe highlighting Chrs. I and III. Quantification of cell viability and foldchange in $\mathrm{CO}$ frequency was based on $\geq 3$ independent experiments and are presented as means with standard deviations (s.d.)

from the reversal of the D-loop by the helicase activity of Srs2, which is also called anti-crossover activity (see below).

Recent reports demonstrated that a mutation affecting ssDNA binding protein RPA ( $r f a 1-t 33$ ) leads to a defect in BIR that may result from decreased Rad51 loading ${ }^{13}$. Thus, we tested whether similar to rad55 , rfa1-t33 can suppress low viability of $s r s 2 \Delta$. Indeed, viability of rfa1-t33 srs $2 \Delta$ double mutant was much higher when compared to srs $2 \Delta$ (Fig. 4a). Moreover, the 
a

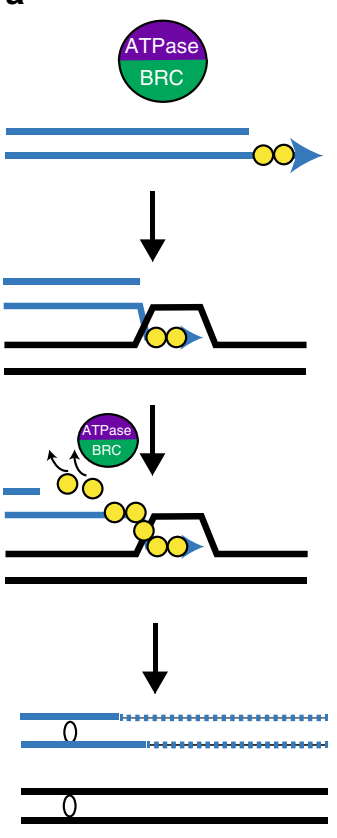

b
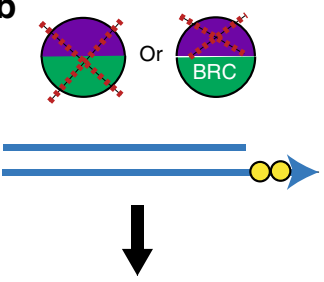
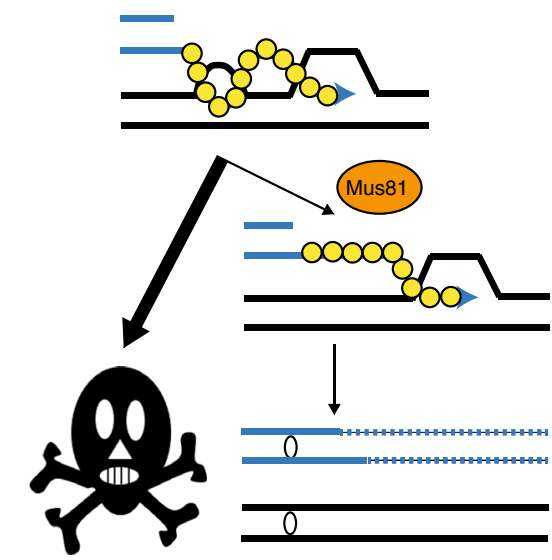

C
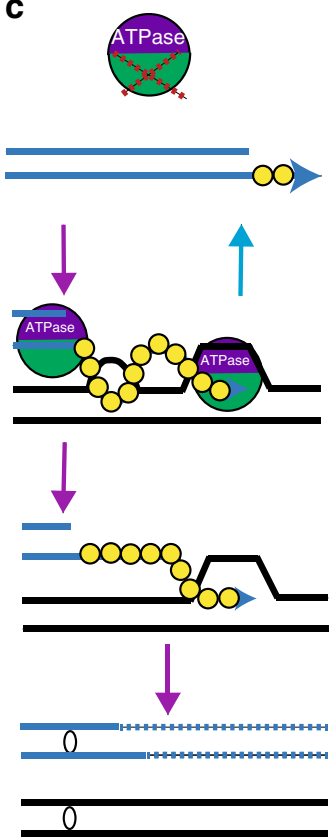

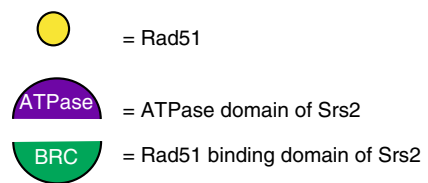

Fig. 6 The model of BIR associated toxic joint molecules. a The strippase activity of Srs2 requiring both the ATPase domain (purple semi-circle) and the Rad51 binding domain (BRC; green) removes excess of Rad51 (yellow circles) from the filament, which prevents formation of toxic intermediates behind the migrating BIR bubble, and ensures efficient BIR. $\mathbf{b}$ In the absence of Srs2 or of its ATPase activity (srs2-K41A) additional Rad51 bound to long ssDNA leads to formation of toxic intermediates, which predominantly lead to cell death (denoted by the skull), but can be resolved by Mus81-Mms4, which allows BIR completion and cell survival. c When strippase activity of Srs2 is defective (in the absence of the Rad51 binding domain), toxic intermediates are accumulated, but can be dismantled by helicase activity of Srs2 (pink arrow). In addition, Srs2 can unwind D-loops using its helicase activity (blue arrow)

proportion of cells completing BIR was significantly increased as compared to rfa1-t33 (Fig. 4b). These results suggested that the defect in the Rad51 filament formation by either rad554 or rfa1t33 suppress the defect of $s r s 2 \Delta$ in BIR. Overall, our results suggest that the need for Srs2 in BIR can be bypassed by compromising the quality ( $r a d 55 \Delta$, rfa1-t33) of Rad51 filament.

The detox role of Srs2 in other DSB induced repair pathways. Having demonstrated the role of Srs2 in counteracting toxic intermediates formed by ssDNA during BIR, we were prompted to ask whether it might play a similar role in other DSB repair pathways including gene conversion and $\mathrm{SSA}^{41}$.

Gene conversion (GC) was tested in a simple ectopic recombination system where DSB induced within a $1.9 \mathrm{~kb} M A T \mathrm{a}$ sequence inserted at the $A R G 5,6$ locus on $\mathrm{Chr}$. $\mathrm{V}$ is repaired by recombination with homologous MATa-inc sequence on Chr. III (Fig. 5a) $)^{40,41}$. Repair in this assay proceeds by SDSA with rare ( $<5 \%$ ) crossover outcomes. It was previously reported that the efficiency of repair, measured $8 \mathrm{~h}$ after DSB by Southern blot analyses of the digested genomic DNA, was decreased in the absence of Srs 2 from $\sim 85 \%$ to about $\sim 30 \%$. Importantly, cell viability following DSB induction in $s r s 2 \Delta$ was reduced to just several percents ${ }^{4}$ (Fig. 5b). In addition, we observed that srs2$B R C \Delta$ did not affect cell viability during GC (Fig. 5b), indicative of the important role of Srs2 motor activity for survival.
As with BIR, the viability of double mutant $\operatorname{srs} 2 \Delta$ rad55 $\Delta$ during ectopic GC was significantly improved as compared to srs $2 \Delta$ or rad55 $\Delta$ single mutants (Fig. $5 \mathrm{~b}$ ). This data suggests that destabilization of Rad51 filament by rad55 $\Delta$ improved SDSA repair in $s r s 2 \Delta$. This supported the idea that the detox function of Srs2 is required for the successful completion of gene conversion. While the efficiency of GC in srs $2 \Delta$ rad55 $\Delta$ mutants appears to be much better than in single $s r s 2 \Delta$ or rad55 $\Delta$, crossover outcomes were dramatically increased when compared to single mutants (Fig. 5a, c). Thus, while repair is improved in srs $2 \Delta$ rad55 cells, it comes at the cost of very high level of crossover that is thought to drive genomic instability and loss of heterozygosity. Therefore, our data suggests that similar to what we demonstrated in BIR, the optimum Rad51 filament required for GC needs both Rad55 and Srs 2 to be present. In addition, our data suggests that Srs2 is unlikely to fulfill its anti-crossover function by dislodging Rad51 from the non-invading DSB end ${ }^{39}$ since this model predicted that rad55 $\Delta$ would likely decrease, not increase, the crossover frequency in $\operatorname{srs} 2 \Delta$. Therefore, our results favor the model that Srs2 D-loop unwinding activity can account for its anti-crossover activity $^{36,56}$. As previously reported in a different system, the anti-crossover activity of Srs2 is largely dependent on its recruitment to SUMO-modified $\mathrm{PCNA}^{25}$. Consistently, in this ectopic recombination assay, the level of crossing-over is increased in mutants affecting SUMO-modification of PCNA, including siz1s, pol30-K127R, pol30-K164R, and the pol30-K127R, 
K164R double mutant (Fig. 5c). Importantly, neither these mutants, nor siz1s srs2-BRCA had significant effect on viability (Fig. 5b), indicating that recruitment of Srs2 to recombination intermediates to promote efficient repair and to suppress crossover pathway are distinct. We also note that sizls did not affect cell viability and the level of BIR (Fig. 3b, c), which is expected considering that disruption of the D-loop would rather counteract than help the D-loop migration. In addition, siz1 $\Delta$ srs2-BRCA did not affect cell viability and the level of BIR as well, consistent with PCNA-independent recruitment of Srs2 to toxic intermediates.

We further hypothesized that the absence of the detox function of Srs2 was also responsible for the death that was previously observed in $s r s 2 \Delta$ following DSB repair by SSA. In that system, DSB was introduced by $\mathrm{HO}$ endonuclease into the LEU2 gene on chromosome III and could be repaired by SSA with a direct, partial LEU2 sequence (U2) located $25 \mathrm{~kb}$ away ${ }^{41}$ (Fig. 5d). More recently, it has been proposed that these DSBs could also be repaired by BIR via strand invasion of LEU2 into $U 2^{41}$. The induction of this repair in $s r s 2 \Delta$ resulted in loss of viability in $98 \%$ of cells ${ }^{41}$ (Fig. 5e). Nevertheless, the authors observed an efficient formation of the repair product $6 \mathrm{~h}$ after the DSB detected by Southern blot analysis of the genomic DNA following its restriction digest and separation by gel electrophoresis ${ }^{41}$. We confirmed this finding in our experiments using Acc65I digested genomic DNA obtained from YMV80 and YMV88 (Supplementary Fig. 8). However, when we analyzed the repair in the same cells using CHEF gel electrophoresis, we observed no repaired chromosomes in YMV88 even after $12 \mathrm{~h}$ following DSB induction (Fig. 5f). We propose that despite the initiation of repair, the intact full chromosomes are never formed in $s r s 2 \Delta$ cells. Thus, we propose that Srs2 plays an important detox role during repair in this SSA/BIR system as well. In addition, srs2-BRCA did not affect cell viability following DSB (Fig. 5e), indicative of the important role of Srs2 motor activity for survival.

\section{Discussion}

BIR proceeds by an unusual, migrating bubble DNA synthesis, with conservative inheritance of newly synthesized strands ${ }^{10,11}$, and with asynchrony between the leading and lagging strand synthesis ${ }^{10}$. The latter leads to the formation of large amounts of ssDNA that is bound by RPA for protection, and can also bind Rad51. Our results suggest that unrestricted binding of Rad51 to ssDNA during BIR promotes unscheduled pairing to the homologous chromosome, which leads to the formation of toxic joint molecules that impede BIR and are lethal to the cell. We propose that Srs2 protects cells from these intermediates. According to our model (Fig. 6), the strippase activity of Srs2, which requires the ATPase and Rad51 interaction domains eliminates the excess of Rad51 from ssDNA regions formed during BIR (Fig. 6a, b), and this prevents the formation of toxic joint molecules. However, if they are nevertheless formed, Srs2 helicase activity, requiring ATPase domain, disrupts them (Fig. 6c). The toxic joint molecules formed in the absence of Srs2, can be resolved by Mus81-Mms4 or Yen1 (Fig. 6b), as we documented by showing: (i) higher viability in $\operatorname{srs} 2 \Delta$ cells possessing Mus81 or Yen1 ${ }^{\mathrm{ON}}$, and (ii) the change of the pattern of the branched DNA structures from the "spike" structures in mus $81 \Delta$ srs $2 \Delta$ to the 'rubble' in srs $2 \Delta$ and in $s r s 2 \Delta$ mus $81 \Delta Y E N 1^{O N}$. We envision that the spike represents high molecular weight and highly branched intermediate that corresponds to multi-invasion regions formed behind the bubble (Supplementary Fig. 5). The resolution of some of the branched structures located inside the multi-invasion region by Mus81 and Yen1 leads to the formation of various molecules comprising the rubble intermediates. These multi- invasion intermediates are still branched because they include varying numbers of 4-way and 3-way junctions that still remain unprocessed (see Supplementary Fig. 5 and EM images in Supplementary Figs. 2, 3, and 6), which leads to varying complexity and varying molecular weight of these molecules comprising the rubble intermediates. These structures could also be dynamic and can be either processed further yielding linear molecules and survival, or may participate in re-formation of toxic structures resulting in cell death observed in $70 \%$ of the cases. We propose that the toxicity observed in $s r s 2 \Delta$ mutants result from unprocessed 3-way junctions, 4-way junctions or other aberrant recombination intermediates formed by the ssDNA accumulated during BIR.

The viable BIR outcomes formed in $s r s 2 \Delta$ via Mus81 resolution resemble those in wild-type cells, but are less frequently associated with mutations. This could result from lesser amounts of exposed ssDNA that can accumulate damage in $\operatorname{srs} 2 \Delta$ as compared to wild-type cells due to a significant amount of ssDNA involved in the formation of toxic joint intermediates. Alternatively, it is possible that the mutagenic fraction of BIR is preferentially killed in $s r s 2 \Delta$ mutants because it might form more complex multi-invasion structures. It is also possible that other aspects of BIR progression were affected in BIR survivors arising in the absence of Srs2. Overall, we report an important new risk for the cell that results from accumulation of ssDNA during BIR: it can be channeled into the formation of toxic joint molecules, and the goal of Srs2 is to prevent it from happening.

We propose that the role of Srs2 in removing toxic joint molecules is especially important in diploids where ssDNA regions located behind the D-loop share large regions of homology with homologous chromosomes. Moreover, our data suggests that a similar role might be played by Srs2 even in haploid cells, for example during SSA/BIR between distant repeats in YMV88 or ectopic gene conversion, where long ssDNA regions are formed ${ }^{41}, 46$, but the areas for promiscuous invasion might be more limited. In particular, we observed that repaired chromosomes were virtually undetectable following SSA/BIR in YMV88 despite the successful formation of the initial repair fragments ${ }^{41}$. The failure of the repaired chromosomes to enter the $\mathrm{CHEF}$ gel is likely indicative of branched toxic joint molecules that can be formed by ssDNA regions generated by resection that proceeded outside of the annealing region in the case of SSA. These toxic intermediates could be formed by the invasion of ssDNA at ectopic positions, at locations of the Ty or delta elements, which can explain a low viability following DSB induction in haploid cells. In fact, using the same SSA system, it was demonstrated that long ssDNA region formed in a course of DSB resection contains a delta element that can invade at ectopic positions which modestly decreased cell viability even in the presence of Srs $2^{57}$. It is likely that the absence of Srs 2 can significantly exacerbate this problem. The importance of Srs2, and especially of its helicase activity for ectopic GC, as well as the restoration of cell viability in srs $2 \Delta$ by deleting RAD55 are indicative of an important anti-toxic role of Srs2 during ectopic GC as well. Therefore, we propose that Srs2 is required for the disruption of toxic joint molecules during ectopic GC and SSA, even though we cannot exclude its importance for other aspects of these DSB repair pathways, including successful DNA synthesis ${ }^{44}$ or homology search ${ }^{58}$. In addition, while the DSB repair by BIR and ectopic GC is greatly improved in $s r s 2 \Delta$ rad55 $\Delta$ as compared to $\operatorname{srs} 2 \Delta$ or rad554, it remains significantly compromised. Our results suggest that Rad51 filament formed in the presence of Rad55/Rad57 and Srs2 proteins that stabilize and disrupt the nucleofilament, respectively, ensures optimal DSB repair kinetics, efficiency and prevents crossover outcomes. 
Our study describes the case where successful repair of a DSB by recombination depends on successful elimination of aberrant recombination intermediates by Srs 2 . This function is similar to the "anti-recombination" function that Srs2 was previously proposed to play during S-phase replication ${ }^{14-19,59}$. In particular, multiple studies have demonstrated that Srs2 prevents channeling of ssDNA gaps resulting from spontaneous and induced DNA damage into recombination that can be detrimental to the cell. The main difference though is that "ssDNA gaps" that can be formed during BIR are potentially much bigger as compared to those arising spontaneously, or in other DNA damage situations. Our idea that Srs2 plays the same role during BIR and broadly in DNA damage repair is supported by our observation that Yen 1 ON suppresses both BIR deficiency and MMS sensitivity of $s r s 2 \Delta$. Overall, based on our study we propose a comprehensive, yet simple, model where the same anti-recombination function of Srs2 is required for successful DSB repair, as well as to oppose spontaneous recombination.

Based on the detox role of Srs2 in BIR we predict that Srs2 is also important for the alternative lengthening of telomeres (ALT), a BIR-like pathway responsible for telomere maintenance in $10-15 \%$ of cancers 9 . We predict that the absence of Srs 2 can result in trapping of chromosomes participating in ALT. Therefore, human counterparts of Srs2 may serve as anti-cancer targets in ALT cells. Overall, we propose that long ssDNA formed during BIR can form lethal joint molecules and we envision that this property can be used in the future development of anti-cancer therapy that will specifically target cells undergoing BIR.

\section{Methods \\ Yeast strains and growth conditions. All yeast strains were isogenic to} AM1003 ${ }^{45}$, which is disomic for Chr. III with a genotype as follows: $h m l \Delta:: A D E 1 /$ $h m l \Delta:: A D E 3$ MATa-LEU2-tel/MAT $\alpha$-inc hmrA::HPH FS2 $:: N A T / F S 2$ leu2/leu23112 thr4 ura3-52 ade3::GAL::HO ade1 met13.

AM3110 was derived from AM1003 in two steps. First, multiple copies of TEF1/ $B S D$ were inserted into SNT1 of the MAT $\alpha$-inc containing copy of Chr. III using standard methods. Integration of multiple copies in Chr. III was confirmed by CHEF gel electrophoresis ${ }^{10}$. Second, p304-BrdU cassette was integrated into TRP1 on Chr. IV and the integration was confirmed by PCR ${ }^{10}$. All strains used in BIR assay were derivatives of AM3110. Strains used for the ectopic GC assays are derivatives of tGI354 ${ }^{40}$. Strains used in SSA assay were derivatives of YMV80 41 . Derivatives containing srs2-BRCA were created using delitto perfetto approach where the $p C O R E$ plasmid ${ }^{60}$ was integrated into the BRC region of SRS2 locus on Chr. X using $50 \mathrm{bp}$ flanking homology on both sides. This cassette was replaced using $100 \mathrm{bp}$ primers that are complementary to each other and are homologous to the sequence flanking SRS2 on both sides of the BRC region. This deletion was confirmed by sequencing (Supplementary Table 3). Strains containing srs2:: KANMX, pol30-K127R::KANMX, pol30-K164R::KANMX and siz1::KANMX were constructed using standard methods with PCR-derived KANMX module ${ }^{41}$. Strains containing mus81::ble ${ }^{r}$, srs2::ble and rad55::ble $e^{r}$ were constructed using standard methods with PCR-derived phleomycin-resistant $\left(\mathrm{ble}^{\mathrm{r}}\right)$ cassette $^{61}$ flanked by terminal sequences matching the first and last $80 \mathrm{bp}$ of the open reading frames of MUS81, SRS2 and RAD55 gene respectively. pol30-K127R::KANMX and pol30K164R::KANMX strains were constructed by using standard methods with PCRderived fragments amplified using genomic DNA of corresponding mutant strains ${ }^{62}$.

Growth media contained rich medium yeast extract-peptone-dextrose (YEPD) and synthetic medium specific bases and amino-acids omitted as specified $^{46,47}$. YEP-lactate (YEP-Lac) and YEP-galactose (YEP-Gal) contained 1\% yeast extract and $2 \%$ Bacto peptone media supplemented with $3.7 \%$ lactic acid $(\mathrm{pH}$ $5.5)$ or $2 \%(\mathrm{w} / \mathrm{v})$ galactose, respectively. Cultures were grown at $30^{\circ} \mathrm{C}$.

Physical analysis of DSB repair. The kinetics and efficiency of BIR, ectopic GC and SSA was analyzed by CHEF gel electrophoresis followed by Southern blotting using $10 \times$ saline-sodium citrate (SSC) buffer and Southern hybridization using the following radiolabeled probes: $A D E 1$ and $A D E 3$-specific probes for the analysis of BIR repair product and donor Chr. III, respectively ${ }^{10}$ and $A D E 1$-specific probes for the analysis of Chr. III repaired by SSA ${ }^{45}$. Images were analyzed using a GE typhoon FLA 7000. Crossover frequency was measured by isolating DNA from cells $8 \mathrm{~h}$ and/or $24 \mathrm{~h}$ after induction of $\mathrm{HO}$ endonuclease. Isolated DNA was digested with EcoR1 and separated on a $0.8 \%$ agarose gel followed by Southern blotting and hybridization with radiolabeled probes. A $800 \mathrm{bp}$ MATa fragment was used as a probe ${ }^{40}$. Density of the GC and crossover bands was calculated using BioRad Quantity One software. Two-dimensional (2D) gel electrophoresis analysis was done by extracting genomic DNA following cesium chloride density gradient centrifugation. Prior to 2D electrophoresis, the genomic DNA was digested by KpnI to analyze BIR intermediates at $0 \mathrm{~kb}$, and by BglII for $24 \mathrm{~kb}$ and $85 \mathrm{~kb}$ positions. Digested DNA was first separated on a $0.4 \%$ agarose gel at $55 \mathrm{~V}(1 \mathrm{D})$ and then separated on a $1.2 \%$ agarose gel at $340 \mathrm{~V}(2 \mathrm{D})^{10}$. Following Southern blotting the intermediates at $0 \mathrm{~kb}, 24 \mathrm{~kb}$, and $85 \mathrm{~kb}$ positions were detected by hybridizing with TAF2, PWP2, and CDC39 -specific radiolabeled probes, respectively.

Genetic analysis of DSB repair. Cell viability following DSB induction was determined by plating cells on YEPD and YEP-Gal media and calculated by dividing the number of colony-forming units (CFUs) on YEP-Gal by the number of CFUs on YEPD. A minimum of three plating experiments was performed to calculate the averages and standard deviations for viability. To characterize the DSB repair outcomes, the colonies formed on YEP-Gal plates were replica plated onto appropriate omission media to determine the fraction of DSB repair events with the following phenotypes: $\mathrm{Ade}^{+} \mathrm{Leu}^{+}$(GC), Ade ${ }^{- \text {white }} \mathrm{Leu}^{-}$(HC), Ade ${ }^{-\mathrm{red}} \mathrm{Leu}^{-}$ (CL), and $\mathrm{Ade}^{+} \mathrm{Leu}^{-}(\mathrm{BIR})^{45}$. The rate of $\mathrm{Lys}^{+}$mutagenesis was determined by plating appropriate concentrations on YEPD media and on media omitting lysine before $(0 \mathrm{~h})$ and $7 \mathrm{~h}$ after the addition of galactose $(7 \mathrm{~h})^{47}$. The rate of $\mathrm{Ura}^{+}$ mutagenesis was determined similar to the $\mathrm{Lys}^{+}$mutagenesis, except that appropriate concentration of cells were plated on YEPD media and on media omitting uracil before $(0 \mathrm{~h})$ and after $(7 \mathrm{~h})$ DSB induction ${ }^{10}$. The rate of mutations after galactose treatment was determined using a simplified version of the Drake equation. This modification was necessary because experimental strains did not divide or underwent one division between $0 \mathrm{~h}$ and $7 \mathrm{~h}^{47}$.

Analysis of BIR intermediates by electron microscopy. EM analysis of BIR intermediates was by collecting samples $7 \mathrm{~h}$ following DSB induction and crosslinked using psoralen to preserve branched intermediates ${ }^{10}$. For No-DSB control, $0.015 \mathrm{mg} / \mathrm{ml}$ nocodazole was added simultaneously with $2 \%$ galactose, and the G2arrested cells were collected $4 \mathrm{~h}$ later. The genomic DNA was extracted and processed similar to the samples in $2 \mathrm{D}$ gel electrophoresis ${ }^{10}$, and digested with BgIII. Following enrichment using BND cellulose ${ }^{63}$, EM samples were prepared by spreading the DNA on carbon-coated grids in the presence of benzyl-dimethylalkylammonium chloride and visualized by platinum rotary shadowing ${ }^{63}$. Images were acquired on a transmission electron microscope (JEOL 1200 EX) with sidemounted camera (AMTXR41 supported by AMT software v601) and analyzed with ImageJ (National Institutes of Health).

Data Availability. All relevant data are available from the authors upon request.

Received: 23 May 2017 Accepted: 27 October 2017

Published online: 27 November 2017

\section{References}

1. Sakofsky, C. J. et al. Break-induced replication is a source of mutation clusters underlying kataegis. Cell Rep. 7, 1640-1648 (2014).

2. Sakofsky, C. J. et al. Translesion polymerases drive microhomology-mediated break-induced replication leading to complex chromosomal rearrangements. Mol. Cell. 60, 860-872 (2015).

3. Vasan, S., Deem, A., Ramakrishnan, S., Argueso, J. L. \& Malkova, A. Cascades of genetic instability resulting from compromised break-induced replication. PLoS Genet. 10, e1004119 (2014).

4. Smith, C. E., Llorente, B. \& Symington, L. S. Template switching during breakinduced replication. Nature 447, 102-105 (2007).

5. Smith, C. E., Lam, A. F. \& Symington, L. S. Aberrant double-strand break repair resulting in half crossovers in mutants defective for Rad51 or the DNA polymerase delta complex. Mol. Cell. Biol. 29, 1432-1441 (2009).

6. Anand, R. P. et al. Chromosome rearrangements via template switching between diverged repeated sequences. Genes Dev. 28, 2394-2406 (2014)

7. Costantino, L. et al. Break-induced replication repair of damaged forks induces genomic duplications in human cells. Science 343, 88-91 (2014).

8. Minocherhomji, S. et al. Replication stress activates DNA repair synthesis in mitosis. Nature 528, 286-290 (2015).

9. Dilley, R. L. \& Greenberg, R. A. Alternative telomere maintenance and cancer Trends Cancer 1, 145-156 (2015).

10. Saini, N. et al. Migrating bubble during break-induced replication drives conservative DNA synthesis. Nature 502, 389-392 (2013)

11. Donnianni, R. A. \& Symington, L. S. Break-induced replication occurs by conservative DNA synthesis. Proc. Natl Acad. Sci. USA 110, 13475-13480 (2013). 
12. Wilson, M. A. et al. Pif1 helicase and Poldelta promote recombination-coupled DNA synthesis via bubble migration. Nature 502, 393-396 (2013).

13. Ruff, P., Donnianni, R. A., Glancy, E., Oh, J. \& Symington, L. S. RPA stabilization of single-stranded DNA is critical for break-induced replication. Cell Rep. 17, 3359-3368 (2016).

14. Aguilera, A. \& Klein, H. L. Genetic control of intrachromosomal recombination in Saccharomyces cerevisiae. I. Isolation and genetic characterization of hyperrecombination mutations. Genetics 119, 779-790 (1988).

15. Aboussekhra, A. et al. RADH, a gene of Saccharomyces cerevisiae encoding a putative DNA helicase involved in DNA repair. Characteristics of radH mutants and sequence of the gene. Nucleic Acids Res. 17, 7211-7219 (1989).

16. Gangloff, S., Soustelle, C. \& Fabre, F. Homologous recombination is responsible for cell death in the absence of the Sgs1 and Srs2 helicases. Nat. Genet. 25, 192-194 (2000).

17. Klein, H. L. Mutations in recombinational repair and in checkpoint control genes suppress the lethal combination of srs2Delta with other DNA repair genes in Saccharomyces cerevisiae. Genetics 157, 557-565 (2001).

18. Veaute, X. et al. The Srs2 helicase prevents recombination by disrupting Rad51 nucleoprotein filaments. Nature 423, 309-312 (2003).

19. Krejci, L. et al. DNA helicase Srs2 disrupts the Rad51 presynaptic filament. Nature 423, 305-309 (2003).

20. Rong, L., Palladino, F., Aguilera, A. \& Klein, H. L. The hyper-gene conversion hpr5-1 mutation of Saccharomyces cerevisiae is an allele of the SRS2/RADH gene. Genetics 127, 75-85 (1991).

21. Aboussekhra, A., Chanet, R., Adjiri, A. \& Fabre, F. Semidominant suppressors of Srs2 helicase mutations of Saccharomyces cerevisiae map in the RAD51 gene, whose sequence predicts a protein with similarities to procaryotic RecA proteins. Mol. Cell Biol. 12, 3224-3234 (1992).

22. Barbour, L. \& Xiao, W. Regulation of alternative replication bypass pathways at stalled replication forks and its effects on genome stability: a yeast model. Mutat. Res. 532, 137-155 (2003).

23. Watts, F. Z. The role of SUMO in chromosome segregation. Chromosoma 116, 15-20 (2007).

24. Lambert, S. et al. Homologous recombination restarts blocked replication forks at the expense of genome rearrangements by template exchange. Mol. Cell 39, 346-359 (2010)

25. Robert, T., Dervins, D., Fabre, F. \& Gangloff, S. Mrc1 and Srs2 are major actors in the regulation of spontaneous crossover. EMBO J. 25, 2837-2846 (2006).

26. Le Breton, C. et al. Srs2 removes deadly recombination intermediates independently of its interaction with SUMO-modified PCNA. Nucleic Acids Res. 36, 4964-4974 (2008).

27. Burgess, R. C. et al. Localization of recombination proteins and Srs2 reveals anti-recombinase function in vivo. J. Cell Biol. 185, 969-981 (2009).

28. Kerrest, A. et al. SRS2 and SGS1 prevent chromosomal breaks and stabilize triplet repeats by restraining recombination. Nat. Struct. Mol. Biol. 16, 159-167 (2009).

29. Urulangodi, M. et al. Local regulation of the Srs2 helicase by the SUMO-like domain protein Esc2 promotes recombination at sites of stalled replication. Genes Dev. 29, 2067-2080 (2015).

30. Liu, J. et al. Rad51 paralogues Rad55-Rad57 balance the antirecombinase Srs2 in Rad51 filament formation. Nature 479, 245-248 (2011).

31. Heude, M., Chanet, R. \& Fabre, F. Regulation of the Saccharomyces cerevisiae Srs2 helicase during the mitotic cell cycle, meiosis and after irradiation. Mol. Gen. Genet. 248, 59-68 (1995)

32. Morel, P., Hejna, J. A., Ehrlich, S. D. \& Cassuto, E. Antipairing and strand transferase activities of E. coli helicase II (UvrD). Nucleic Acids Res. 21, 3205-3209 (1993).

33. Krejci, L. et al. Role of ATP hydrolysis in the antirecombinase function of Saccharomyces cerevisiae Srs2 protein. J. Biol. Chem. 279, 23193-23199 (2004).

34. Colavito, S. et al. Functional significance of the Rad51-Srs2 complex in Rad51 presynaptic filament disruption. Nucleic Acids Res. 37, 6754-6764 (2009).

35. Marini, V. \& Krejci, L. Unwinding of synthetic replication and recombination substrates by Srs2. DNA Repair 11, 789-798 (2012).

36. Liu, J. et al. Srs2 promotes synthesis-dependent strand annealing by disrupting DNA polymerase delta extending D-loops. eLife https://doi.org/10.7554/eLife 22195 (2017).

37. Potenski, C. J., Niu, H., Sung, P. \& Klein, H. L. Avoidance of ribonucleotideinduced mutations by RNase $\mathrm{H} 2$ and Srs2-Exol mechanisms. Nature 511, 251-254 (2014)

38. Bhattacharyya, S. \& Lahue, R. S. Saccharomyces cerevisiae Srs2 DNA helicase selectively blocks expansions of trinucleotide repeats. Mol. Cell Biol. 24, 7324-7330 (2004)

39. Mitchel, K., Lehner, K. \& Jinks-Robertson, S. Heteroduplex DNA position defines the roles of the Sgs1, Srs2, and Mph1 helicases in promoting distinct recombination outcomes. PLoS Genet. 9, e1003340 (2013).

40. Ira, G., Malkova, A., Liberi, G., Foiani, M. \& Haber, J. E. Srs2 and Sgs1-Top3 suppress crossovers during double-strand break repair in yeast. Cell 115, 401-411 (2003).
41. Vaze, M. B. et al. Recovery from checkpoint-mediated arrest after repair of a double-strand break requires Srs2 helicase. Mol. Cell 10, 373-385 (2002).

42. Lydeard, J. R. et al. Break-induced replication requires all essential DNA replication factors except those specific for pre-RC assembly. Genes Dev. 24, 1133-1144 (2010).

43. Yeung, M. \& Durocher, D. Srs2 enables checkpoint recovery by promoting disassembly of DNA damage foci from chromatin. DNA Repair. 10, 1213-1222 (2011).

44. Vasianovich, Y. et al. Unloading of homologous recombination factors is required for restoring double-stranded DNA at damage repair loci. EMBO J. 36, 213-231 (2017).

45. Deem, A. et al. Defective break-induced replication leads to half-crossovers in Saccharomyces cerevisiae. Genetics 179, 1845-1860 (2008).

46. Chung, W. H., Zhu, Z., Papusha, A., Malkova, A. \& Ira, G. Defective resection at DNA double-strand breaks leads to de novo telomere formation and enhances gene targeting. PLoS Genet. 6, e1000948 (2010).

47. Deem, A. et al. Break-induced replication is highly inaccurate. PLoS Biol. 9, e1000594 (2011).

48. Malkova, A., Ivanov, E. L. \& Haber, J. E. Double-strand break repair in the absence of RAD51 in yeast: a possible role for break-induced DNA replication. Proc. Natl Acad. Sci. USA 93, 7131-7136 (1996).

49. Schwartz, E. K. \& Heyer, W. D. Processing of joint molecule intermediates by structure-selective endonucleases during homologous recombination in eukaryotes. Chromosoma 120, 109-127 (2011).

50. Hunter, N. \& Kleckner, N. The single-end invasion: an asymmetric intermediate at the double-strand break to double-holliday junction transition of meiotic recombination. Cell 106, 59-70 (2001).

51. Schwacha, A. \& Kleckner, N. Identification of joint molecules that form frequently between homologs but rarely between sister chromatids during yeast meiosis. Cell 76, 51-63 (1994).

52. Schwacha, A. \& Kleckner, N. Identification of double Holliday junctions as intermediates in meiotic recombination. Cell 83, 783-791 (1995).

53. Blanco, M. G., Matos, J. \& West, S. C. Dual control of Yen1 nuclease activity and cellular localization by Cdk and Cdc14 prevents genome instability. Mol. Cell 54, 94-106 (2014).

54. Eissler, C. L. et al. The Cdk/cDc14 module controls activation of the Yen1 holliday junction resolvase to promote genome stability. Mol. Cell 54, 80-93 (2014).

55. Niu, H. \& Klein, H. L. Multifunctional roles of Saccharomyces cerevisiae Srs2 protein in replication, recombination and repair. FEMS Yeast Res. 17, Fow111, https://doi.org/10.1093/femsyr/fow111 (2017).

56. Dupaigne, P. et al. The Srs2 helicase activity is stimulated by Rad51 filaments on dsDNA: implications for crossover incidence during mitotic recombination. Mol. Cell 29, 243-254 (2008).

57. Jain, S., Sugawara, N. \& Haber, J. E. Role of double-strand break end-tethering during gene conversion in Saccharomyces cerevisiae. PLoS Genet. 12, e1005976 (2016).

58. Houston, P. L. \& Broach, J. R. The dynamics of homologous pairing during mating type interconversion in budding yeast. PLoS Genet. 2, e98 (2006).

59. Keyamura, K., Arai, K. \& Hishida, T. Srs2 and Mus81-Mms4 prevent accumulation of toxic inter-homolog recombination intermediates. PLoS Genet. 12, e1006136 (2016).

60. Storici, F. \& Resnick, M. A. Delitto perfetto targeted mutagenesis in yeast with oligonucleotides. Genet. Eng. 25, 189-207 (2003).

61. Gueldener, U., Heinisch, J., Koehler, G. J., Voss, D. \& Hegemann, J. H. A second set of loxP marker cassettes for Cre-mediated multiple gene knockouts in budding yeast. Nucleic Acids Res. 30, e23 (2002).

62. Pfander, B., Moldovan, G. L., Sacher, M., Hoege, C. \& Jentsch, S. SUMOmodified PCNA recruits Srs2 to prevent recombination during $\mathrm{S}$ phase. Nature 436, 428-433 (2005).

63. Neelsen, K. J., Chaudhuri, A. R., Follonier, C., Herrador, R. \& Lopes, M. Visualization and interpretation of eukaryotic DNA replication intermediates in vivo by electron microscopy. Methods Mol. Biol. 1094, 177-208 (2014).

\section{Acknowledgements}

We thank Dr. Miguel Blanco for providing YEN1 ${ }^{O N}$ plasmid. We thank Dr. Hannah Klein for providing srs2-K41A construct. We are thankful to Dr. Stefan Jentsch for the gift of pol30 mutant strains. We thank Drs. James Haber, Wolf-Dietrich Heyer, and Michael Lichten for helpful discussions. The work was funded by NIH grants R01GM084242 to A.M., GM080600 and CPRIT RP140456 to G.I., NIH grant R01GM108648 and by DOD BRCP Breakthrough Award BC151728 to A.V.

\section{Author contributions}

R.E., Z.S., K.L, G.I, A.V., and A.M. designed the experiments. R.E., J.D., N.P., D.L. constructed strains. R.E., J.D., C.S., Y.I., N.P., D.L., G.I. performed the BIR, ectopic GC and SSA experiments. Z.S., R.E., and K.L. performed 2D gel electrophoresis. J.J., R.E., A. 
V. performed EM experiments. R.E., G.I, and A.M. wrote the manuscript. R.E. and Z.S. contributed equally to this work.

\section{Additional information}

Supplementary Information accompanies this paper at https://doi.org/10.1038/s41467017-01987-2.

Competing interests: The authors declare no competing financial interests.

Reprints and permission information is available online at http://npg.nature.com/ reprintsandpermissions/

Publisher's note: Springer Nature remains neutral with regard to jurisdictional claims in published maps and institutional affiliations. (c) (i) Open Access This article is licensed under a Creative Commons Attribution 4.0 International License, which permits use, sharing, adaptation, distribution and reproduction in any medium or format, as long as you give appropriate credit to the original author(s) and the source, provide a link to the Creative Commons license, and indicate if changes were made. The images or other third party material in this article are included in the article's Creative Commons license, unless indicated otherwise in a credit line to the material. If material is not included in the article's Creative Commons license and your intended use is not permitted by statutory regulation or exceeds the permitted use, you will need to obtain permission directly from the copyright holder. To view a copy of this license, visit http://creativecommons.org/ licenses/by/4.0/.

(C) The Author(s) 2017 